\title{
Good COP1 or bad COP1? In vivo veritas
}

\author{
Wenyi Wei ${ }^{1}$ and William G. Kaelin Jr. ${ }^{2,3}$ \\ 1Department of Pathology, Beth Israel Deaconess Medical Center, Harvard Medical School, Boston, Massachusetts, USA. ${ }^{2}$ Department of Medical Oncology, \\ Dana-Farber Cancer Institute, Harvard Medical School, Boston, Massachusetts, USA. ${ }^{3}$ Howard Hughes Medical Institute, Chevy Chase, Maryland, USA.
}

\begin{abstract}
The evolutionarily conserved protein COP1 has been shown to operate as an E3 ubiquitin ligase complex, and a number of putative substrates have been identified, including the c-JUN oncoprotein and p53 tumor suppressor protein. New work by Migliorini and colleagues described in the current issue of JCI demonstrates that COP1 acts as a tumor suppressor in vivo and does so, at least in part, by promoting the destruction of $\mathrm{c}-J U N$. These findings challenge the view that COP1 regulates p53 stability and call into question the wisdom of developing COP1 inhibitors as potential anticancer agents.
\end{abstract}

Ubiquitin ligase complexes that target proteins for proteasomal degradation in a regulated manner play important roles in maintaining cellular homeostasis (1). Accordingly, deregulation of ubiquitin ligases and their protein targets has been implicated in a variety of diseases including cancer. For example, overexpression of MDM2, which ubiquitinates the p53 tumor suppressor protein, has been linked to certain cancers, as has loss of AXIN, which is a component of a ubiquitin ligase that targets the $\beta$-catenin oncoprotein for destruction. In this issue of JCI, Migliorini et al. provide evidence that loss of constitutive photomorphogenesis protein 1 (Cop1), a component of another ubiquitin ligase complex, leads to deregulation of the c-Jun oncoprotein and tumor growth in vivo.

COP1, also called RFW2 (RING finger and WD repeat domain 2), was initially identified in Arabidopsis, where it plays a critical role in growth and development in response to light (photomorphogenesis) (2). Under dark conditions, COP1 represses photomorphogenesis by serving as an E3 ubiquitin ligase that targets proteins such as the photomorphogenesis-promoting transcription factor HY5 for destruction (3). In response to light, COP1 becomes inactive, both by virtue of translocation out of the nucleus and through inhibitory protein-protein interactions, thereby allowing photomorphogenesis to proceed.

\section{COP1 is an ubiquitin ligase}

Although mammalian cells do not undergo photomorphogenesis, they somewhat sur-

Conflict of interest: The authors have declared that no conflict of interest exists.

Citation for this article: J Clin Invest doi:10.1172/ JCI57080. prisingly also contain an Arabidopsis COP1 homolog. Mammalian COP1, like the Arabidopsis protein, consists of an $\mathrm{N}$-terminal RING finger domain, an internal coiledcoil domain, and C-terminal WD40 repeats. Moreover, mammalian COP1 possesses E3 ubiquitin ligase activity (4). In addition to possessing intrinsic E3 activity, COP1 participates, in both Arabidopsis and mammalian cells, in a higher-order E3 ubiquitin ligase complex that contains DET1 (deetiolated 1), DDB1 (DNA damage-binding protein 1), CUL4 (cullin 4), and RBX1 (ring-box 1) (4). The growing list of COP1 substrates identified so far includes $\mathrm{p} 53$ (5), c-JUN (6, 7), ACC1 (acetyl-coenzyme A carboxylase alpha) (8), MTA1 (metastasisassociated protein 1) (9), FOXO1 (forkhead box protein O1) (10), TORC2 (transducer of regulated CREB activity 2) (11), and PEA3 (polyomavirus enhancer activator-3) (12). The evidence for these being COP1 targets rests entirely on biochemical and cell culture experiments, raising questions as to which are actually regulated by $\mathrm{COP} 1$ in vivo. Moreover, inclusion of the p53 tumor suppressor protein and the c-JUN oncoprotein as potential COP1 targets made it difficult to predict whether COP1 would serve primarily as an oncoprotein or as a tumor suppressor (Figure 1), despite recent calls to develop COP1 inhibitors for use as cancer therapeutics.

\section{Cop1 regulates c-Jun and functions as a tumor suppressor in vivo}

To more fully understand the physiological roles of COP1, Migliorini and colleagues utilized a genetic approach to generate an allelic series of Cop1-mutant mice (13). The use of hypomorphic (partial-loss-of-function) alleles is a potentially powerful way to circumvent lethality associated with null alleles and also allows one to interrogate the doseresponse relationships linking gene activity to functional outputs. The methodology employed by Migliorini and coworkers can be used with genes that have been targeted with pGT0lxf or pGT0lxr vectors, available through the International Gene Trap Consortium (http://www.genetrap.org).

The authors found that c-Jun abundance, but not p53 abundance or activity, was elevated in Cop1-hypomorphic mice that expressed only $10 \%$ of Cop 1 compared with the wild-type counterparts (13). Increased c-Jun accumulation was shown to contribute to the increased proliferation manifest by Cop1-defective cells in vitro. More importantly, Cop1-hypomorphic mice developed spontaneous malignancy and were more prone to radiation-induced lymphomagenesis. The study therefore firmly establishes that Cop1 is an ubiquitin ligase for c-Jun and that Cop1 acts as a tumor suppressor in vivo. In keeping with the latter, the authors found that COP1 mRNA levels are diminished in certain human cancers, such as prostate cancer, and that restoration of COP1 function in COP1-defective human prostate cancer suppressed their proliferation in vitro. In contrast, the current study found no evidence that Cop 1 regulates p53 in vivo, although it remains possible that Cop 1 serves as a 53 ubiquitin ligase under specific conditions other than those tested in their model.

\section{c-JUN stability is governed by multiple E3 ubiquitin ligases}

c-JUN is the founding member of the AP-1 (activating protein-1) family of transcription factors, which bind to TRE/AP-1 elements $\left(5^{\prime}\right.$-TGA[CG]TCA-3') in their target genes (14). By forming heterodimers with other AP-1 family members (including JUNB, JUND, c-FOS, and ATF), c-JUN participates in a variety of cellular functions including the regulation of cell cycle progression, cell growth, and apoptosis. As is true for many critical transcription factors, including c-MYC and p53, c-JUN is a highly unstable protein (15) and is subject to regulation by multiple ubiquitin ligases (16). In addition to COP1, both FBW7 (F-box/WD 
A Model I

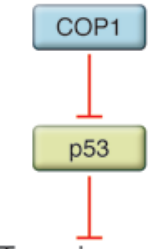

Tumorigenesis
B Model II

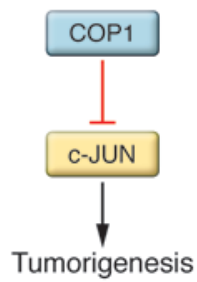

Figure 1

COP1 functions as an E3 ubiquitin ligase. Both p53 and c-JUN have been identified as potential COP1 substrates. Theoretically, COP1 could act as an oncoprotein by promoting the destruction of the p53 tumor suppressor (A; Model I) or as a tumor suppressor protein by promoting the destruction of C-JUN (B; Model II). Data provided in the current issue of $\mathrm{JCl}$ by Migliorini et al., obtained using genetically engineered mice, strongly favor Model II.

repeat-containing protein 7) (17) and ITCH (18) (so named because of the dermatitis seen in mice lacking the murine homolog) have been shown to promote c-JUN ubiquitination (Figure 2). c-JUN exists in both unphosphorylated and phosphorylated forms. The JNK kinases phosphorylate, and thereby activate, c-JUN. On the other hand, phosphorylation of ITCH by JNK stimulates ITCH ubiquitin ligase activity (18), thereby establishing a potential negative feedback loop. Whether the net effect of JNK on c-JUN stability is positive or negative, however, is controversial and may be context dependent. Recognition of c-JUN by FBW7 requires that c-JUN first be phosphorylated on Thr239 and Ser243 (17). The Thr239 site is believed to be phosphorylated by GSK3, thereby providing a link to signaling by PI3K and AKT. On the other hand, COP1 recognizes a highly conserved degron in c-JUN that is distinct from the sites recognized by ITCH and FBW7 and which does not appear to depend on phosphorylation (Figure 2).

\section{C-JUN and cancer}

Aberrant c-JUN activity has been well documented in various types of cancers. For example, some cancers overproduce c-JUN by virtue of $c$-JUN amplification (19). Interestingly, the viral counterpart of c-JUN, $\mathrm{v}$-Jun, harbors a missense mutation that allows it to escape degradation by FBW7, leading to its increased stability. FBW7 is itself frequently deleted in many cancers. p53 loss, which occurs commonly in human cancer, leads to JNK activation and a subsequent increase in c-JUN transcription activity. Indeed, Migliorini and coworkers exploited this fact to reveal the deleterious consequences of COP1 loss on cell proliferation in vitro.

\section{Looking forward}

Although c-JUN inactivation abrogated the increased proliferation observed in COP1defective cells in vitro, it is apparently not sufficient to blunt tumorigenesis in Cop1defective mice. This raises the question as to which other COP1 targets conspire with deregulated c-JUN to promote tumor growth in vivo. Other putative COP1 targets that are suspected or known to act as oncogenes include ACC1, PEA3, MTA1, and TORC2 (Figure 2). A more thorough examination of the degree to which COP1 activity is compromised in human cancer would also be timely.

Arabidopsis COP1 activity is exquisitely sensitive to light. It will be important to determine the signal, or signals, that regulate the destruction of c-JUN by mammalian COP1. Presumably those signals will impinge on COP1 itself, c-JUN, or both. In this regard, mammalian COP1 seems to be a target of the DNA damage-responsive ATM kinase, which triggers COP1 auto-ubiquitination and destruction (20). Mammalian COP1, like Arabidopsis COP1, is located in both the nucleus and cytoplasm. It will be of interest to determine whether the subcellular localization of mammalian COP1, like that of its Arabidopsis counterpart (2), is altered in response to specific signals. Answers to these questions should reveal how an ancient developmental program that renders plants sensitive to light has been adapted for use in mammalian cells.

\section{Acknowledgments}

W. Wei is a Massachusetts Life Sciences Center New Investigator and Department of Defense Prostate Research Program New Investigator (PC080377). W. Wei is also supported by NIH grant GM089763. W.G. Kaelin Jr. is a Howard Hughes Investigator

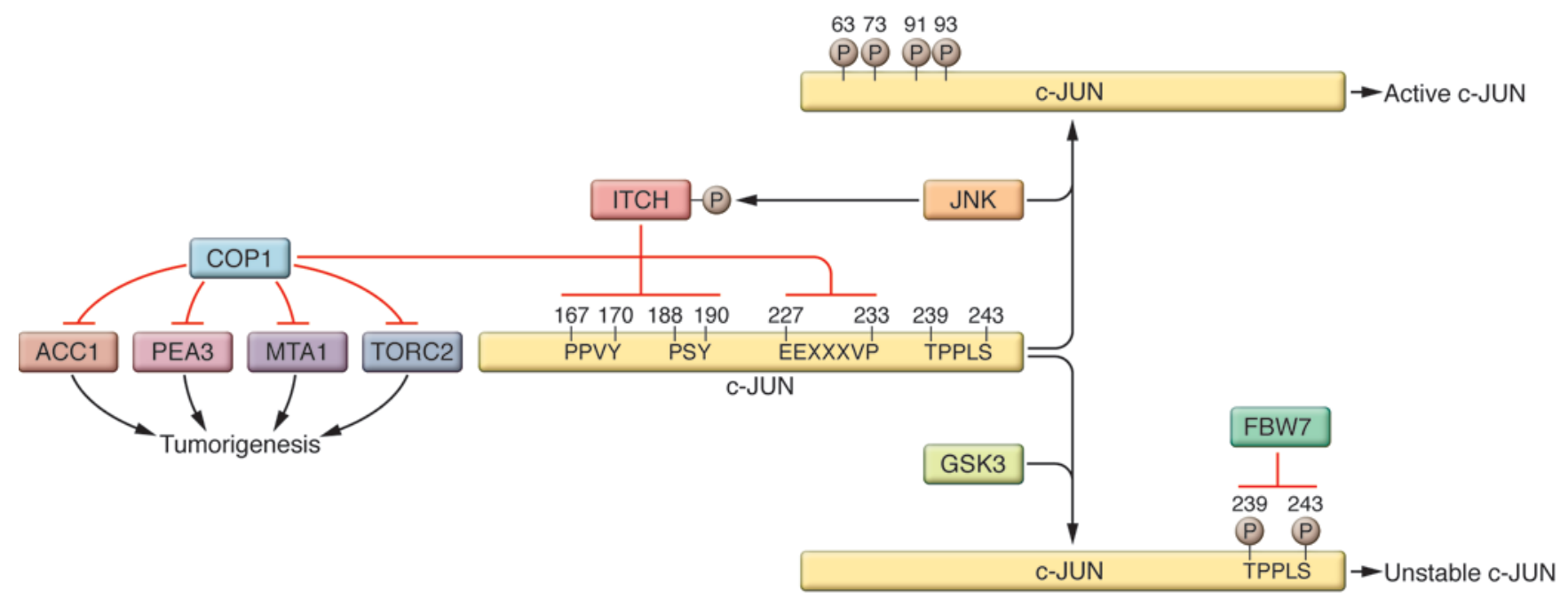

\section{Figure 2}

$\mathrm{COP1}$, ITCH, and FBW7 regulate C-JUN stability. Each of these proteins recognizes a distinct degron within c-JUN. Regulation of c-JUN by ITCH and FBW7 is responsive to changes in JNK and GSK3 activity, respectively. In addition to c-JUN, COP1 has other substrates that might contribute to tumor formation in COP1-defective tissues. 
and a Doris Duke Distinguished Clinical Scientist, and is supported by NIH grants CA068490 and CA101942.

Address correspondence to: William G. Kaelin Jr., Department of Medical Oncology, Dana-Farber Cancer Institute, Harvard Medical School, 450 Brookline Avenue, Mayer 457, Boston, Massachusetts 02115, USA. Phone: 617.632.3975; Fax: 617.632.4760; E-mail: William_Kaelin@ dfci.harvard.edu.

1. Deshaies RJ, Joazeiro CA. RING domain E3 ubiquitin ligases. Annu Rev Biochem. 2009;78:399-434.

2. Schwechheimer C, Deng XW. The COP/DET/FUS proteins-regulators of eukaryotic growth and development. Semin Cell Dev Biol. 2000;11(6):495-503.

3. Osterlund MT, Hardtke CS, Wei N, Deng XW. Targeted destabilization of HY5 during light-regulated development of Arabidopsis. Nature. 2000; 405(6785):462-466.

4. Yi C, Deng XW. COP1 - from plant photomorphogenesis to mammalian tumorigenesis. Trends Cell
Biol. 2005;15(11):618-625

5 . Dornan D, et al. The ubiquitin ligase COP1 is a critical negative regulator of p53. Nature. 2004; 429(6987):86-92.

6. Wertz IE, et al. Human De-etiolated-1 regulates c-Jun by assembling a CUL4A ubiquitin ligase. Science. 2004;303(5662):1371-1374.

7. Savio MG, Rotondo G, Maglie S, Rossetti G, Bender JR, Pardi R. COP1D, an alternatively spliced constitutive photomorphogenic-1 (COP1) product, stabilizes UV stress-induced c-Jun through inhibition of fulllength COP1. Oncogene. 2008;27(17):2401-2411.

8. Qi L, et al. TRB3 links the E3 ubiquitin ligase COP1 to lipid metabolism. Science. 2006; 312(5781):1763-1766

9. Li DQ, et al. E3 ubiquitin ligase COP1 regulates the stability and functions of MTA1. Proc Natl Acad Sci US A. 2009;106(41):17493-17498.

10. Kato S, Ding J, Pisck E, Jhala US, Du K. COP1 functions as a FoxO1 ubiquitin E3 ligase to regulate FoxO1-mediated gene expression. J Biol Chem. 2008; 283(51):35464-35473.

11. Dentin R, et al. Insulin modulates gluconeogenesis by inhibition of the coactivator TORC2. Nature. 2007; 449(7160):366-369.

12. Baert JL, Monte D, Verreman K, Degerny C, Coutte $\mathrm{L}$, de Launoit $\mathrm{Y}$. The E3 ubiquitin ligase complex component COP1 regulates PEA3 group member stability and transcriptional activity. Oncogene. 2010; 29(12):1810-1820.

13. Migliorini D, et al. Cop1 constituively regulates c-Jun protein stability and functions as a tumor suppressor in mice. J Clin Invest. doi:10.1172/JCI45784.

14. Shaulian E, Karin M. AP-1 as a regulator of cell life and death. Nat Cell Biol. 2002;4(5):E131-E136.

15. Jariel-Encontre I, et al. Complex mechanisms for c-fos and c-jun degradation. Mol Biol Rep. 1997; 24(1-2):51-56.

16. Westermarck J. Regulation of transcription factor function by targeted protein degradation: an overview focusing on p53, c-Myc, and c-Jun. Methods Mol Biol. 2010;647:31-36.

17. Wei W, Jin J, Schlisio S, Harper JW, Kaelin WG Jr. The v-Jun point mutation allows c-Jun to escape GSK3dependent recognition and destruction by the Fbw7 ubiquitin ligase. Cancer Cell. 2005;8(1):25-33.

18. Gao M, et al. Jun turnover is controlled through JNK-dependent phosphorylation of the E3 ligase Itch. Science. 2004;306(5694):271-275.

19. Mariani O, et al. JUN oncogene amplification and overexpression block adipocytic differentiation in highly aggressive sarcomas. Cancer Cell. 2007; 11(4):361-374.

20. Dornan D, et al. ATM engages autodegradation of the E3 ubiquitin ligase COP1 after DNA damage. Science. 2006;313(5790):1122-1126. 